\title{
Design optimization and fabrication of a novel structural piezoresistive pressure sensor for micro-pressure measurement
}

\author{
Chuang Li $\mathrm{L}^{\mathrm{a}, \mathrm{b}, \square}$, Francisco Cordovilla ${ }^{\mathrm{a}}$, José L. Ocaña ${ }^{\mathrm{a}}$ \\ ${ }^{a}$ UPM Laser Center, Polytechnical University of Madrid, Carretera de Valencia, km. 7.3, 28031 Madrid, Spain \\ ${ }^{\mathrm{b}}$ Suzhou Changfeng Avionics Co., Ltd, 379, Jianlin Road, High-tech District, 215151 Suzhou, PR China
}

\begin{abstract}
This paper presents a novel structural piezoresistive pressure sensor with a four-beams-bossed-membrane (FBBM) structure that consisted of four short beams and a central mass to measure micro-pressure The proposed structure can alleviate the contradiction between sensitivity and linearity to realize the micro measurement with high accuracy In this study, the design, fabrication and test of the sensor are involved By utilizing the finite element analysis (FEA) to analyze the stress distribution of sensitive elements and subsequently deducing the relationships between structural dimensions and mechanical performance, the optimization process makes the sensor achieve a higher sensitivity and a lower pressure nonlinearity Based on the deduced equations, a series of optimized FBBM structure dimensions are ultimately determined The designed sensor is fabricated on a silicon wafer by using traditional MEMS bulk-micromachining and anodic bonding technology Experimental results show that the sensor achieves the sensitivity of $465 \mathrm{mV} / \mathrm{V} / \mathrm{kPa}$ and pressure nonlinearity of $025 \%$ FSS in the operating range of $0-5 \mathrm{kPa}$ at room temperature, indicating that this novel structure sensor can be applied in measuring the absolute micro pressure lower than $5 \mathrm{kPa}$
\end{abstract}

Keywords:

MEMS piezoresistive pressure sensor Four-beams-bossed-membrane

Finite element analysis Sensitivity

Pressure nonlinearity

\section{Introduction}

The MEMS piezoresistive pressure sensors have been well developed and widely used in the industrial and commercial applications with the vigorous development of silicon micromachining technology $[1,2]$. Recently, with the market expansion of electronic devices including automobiles, aerospace and portable electronics, great research effects have been motivated again to develop high accuracy and micro pres sure measurement sensors which feature high reliability, low costs and mass fabrication capability [3 5]. The key principle of the sensor is based on a thin membrane that deflects when a pressure is applied on the membrane surface. A Wheatstone bridge is built to transduce the resistance change to voltage by means of the piezoresistive effect [6].

During the past years, several typical membrane structures of pie zoresistive pressure sensors have been developed. As it is known, the structure of the membrane plays an important role in the performance of the sensor. $\mathrm{C}$ type membrane, which formed by a cavity on the si licon wafer like the alphabet " $\mathrm{C}$ " from the side face, was the first pro posed structure and was widely used for measuring the pressure of gas or water $[7,8]$. However, a thin $C$ type membrane utilized in the low pressure measurement usually results in a dramatic drop in the me chanical nonlinearity error. Severe pressure nonlinearity $(P N L)$ may cause a high sensitivity device of little practical value once it is beyond a certain range [9]. To solve this problem, the E type membrane structure featured a central island mass was introduced, but this structure sacrificed the sensitivity of the sensor because of the stiffening of the membrane [10]. Sensitivity is proportional to the (membrane length)/(membrane thickness) ratio $(L / H)$, so it can be increased by a larger ratio of that quantity. Unfortunately, the pressure nonlinearity increases with this ratio at a much faster rate, as the pressure non linearity of the pressure to stress conversion is proportional to $(L / H)^{4}$ [11]. Thus, the contradiction between sensitivity and linearity is always irreconcilable for traditional membrane structure. In order to improve the accuracy of the sensor, namely, obtain high sensitivity and low pressure nonlinearity synchronously, previous efforts were mainly fo cused on the following three aspects.

For the first aspect, design a novel structure and create stress con centration regions (SCRs) on the surface of the membrane. All the piezoresistors can be placed on the SCRs and the piezoresistive sensi tivity can be improved with a small deflection of the membrane. For example, Yu et al. [12] developed a beams membrane mono island (BMMI) structure to create SCRs and localize more strain energy within a relatively narrow space. The sensor achieved a high sensitivity of $11.098 \mu \mathrm{V} / \mathrm{V} / \mathrm{Pa}$ in the operating range of $500 \mathrm{~Pa}$ at room temperature,

\footnotetext{
* Corresponding author at: UPM Laser Center, Polytechnical University of Madrid, Carretera de Valencia, km 73 , 28031 Madrid, Spain

E-mail address: chuang li01@alumnos upm es (C Li)
} 
although its pressure nonlinearity of $3.046 \%$ FSS was a little high. Tian et al. [13] designed a novel micro pressure sensor with a cross beam membrane (CBM) structure to enhance stress concentration on the sensitive beams. The sensor obtained a fine sensitivity of $7 \mathrm{mV} / \mathrm{kPa}$ and a low pressure nonlinearity of $0.19 \%$ FSS within the range of $010 \mathrm{kPa}$, which achieved a high accuracy of the sensor due to the creation of SCRs.

For the second aspect, an alternative option is to stiffen the mem brane of the sensor and, consequently, reduce its deflection. According to the definition of the pressure nonlinearity [14], a small membrane deflection will lead to a drop in the gradient of the output curve, thereby reducing the difference between the real output and ideal linear output is an effective way to improve linearity. Kinnella et al. [15] presented a membrane with a thin walled hollow stiffening structure to lessen the 'balloon effect' [16], getting a pressure nonlinearity of less than $0.40 \%$ FSS. H. Sandmaier et al. [17] proposed a sensor chip structure based on a narrow mass in the center of the membrane to make the membrane become harder and decrease its deflection. A lower pressure nonlinearity of $0.05 \%$ FSS was obtained, but the sensitivity was only $3.5 \mu \mathrm{V} / \mathrm{V} / \mathrm{Pa}$ for the measurement of $10 \mathrm{kPa}$.

Finally, many new raw materials for the membrane were developed to improve the overall performance of the sensor. For instance, Pramanik et al. reported that nanocrystalline silicon was used as active piezoresistors for achieving high accuracy [18]. For high precision applications, ceramic based substrate has also been chosen to fabricate the sensor [19]. Some other novel materials, such as polysilicon [20], SiC [21], diamond [22] and silicon nanowires [23] were all adopted to fabricate sensor chips. Although the performance of those pressure sensors have been improved a lot because of these new materials, the performance still cannot satisfy the requirement of achieving high sensitivity and low pressure nonlinearity at the same time. Besides, there are still many practical difficulties in bulk production because of the technical problems.

In consideration of all the advantages and disadvantages for above models, it is found that creating SCRs are beneficial to utilize the strain energy for the piezoresistors, then the sensitivity can be improved de finitely. Also, stiffening the membrane can avoid a large deformation, and then the pressure nonlinearity is expected to control over a small range. The objective of this paper is to design a novel structural membrane combining the advantages of these two types of models mentioned above. New materials are not considered for this paper, as some technical processes are not mature enough for us.

In this study, we reported a novel four beams bossed membrane (FBBM) structural MEMS pressure sensor. By introducing beams into the membrane, the SCRs were expected to be formed. In addition, a central mass not only could play a role in stiffening the membrane, but also improved the performance of the high overload resistance due to the existence of the island to prevent an oversize displacement. This novel sensor was expected to alleviate the contradiction between the sensitivity and linearity and could be used in the measurement of micro pressure. The properties of the sensor, including the mechanical per formance and the relative equations for accurate design and fabrication were fully studied. The fabricated sensor device was tested and com pared with the FEA results, which proved the accuracy of the design process.

\section{Sensor design}

\subsection{Design principle}

For piezoresistive pressure sensors, a square membrane is always used in consideration of its high stress and easy processing when compared with circular and rectangular ones in the same size condition. According to the theory of elasticity, the maximum stresses of the square, rectangular and circular membranes are given by the following equations [24]: $\sigma_{\mathrm{sm}}=0.308 P\left(\frac{L}{H}\right)^{2}\left(1-\mu^{2}\right)$

$\sigma_{\mathrm{rm}}=0.383 P\left(\frac{B}{H}\right)^{2}\left(1-\mu^{2}\right)$

$\sigma_{\mathrm{cm}}=0.75 P\left(\frac{R}{H}\right)^{2}\left(1-\mu^{2}\right)$

where $\sigma_{s m}, \sigma_{r m}$ and $\sigma_{c m}$ are the maximum stresses for the square, rec tangular and circular membranes, respectively. $P$ is the applied pres sure; $H$ is the membrane thickness; $\mu$ is the Poisson ratio; and $L$ is the side length of the square membrane, $B$ is the width of the rectangular membrane, and $R$ is the radius of the circular membrane. By assuming that membrane thickness $H$ and applied load $P$ are totally same for the three models, additionally, $L$ is 1.2 times $B$ and 2 times $R$ respectively, the following relationships can be obtained:

$\sigma_{\mathrm{sm}} \approx 1.16 \sigma_{\mathrm{rm}} \approx 1.64 \sigma_{\mathrm{cm}}$

which means an $\sim 15 \%$ and $\sim 60 \%$ improvement for the stress can be achieved by choosing the square membrane rather than other two types.

According to the small deflection theory, only when the deflection is smaller than $1 / 5$ thickness of the membrane, this theory works, and then the pressure nonlinearity below $1.0 \%$ FSS is possible to obtain and the strain displacement relations are linear [25]. Therefore, keeping the deflection less than $1 / 5$ thickness of the membrane has a positive effect on the linearity of the sensor, and normally, it is possible to obtain high sensitivity and low pressure nonlinearity simultaneously.

The piezoresistive effect has been widely used as the mechanism of the pressure sensor. The resistance of a doped resistor will be changed when the membrane surface experiences a strain or deformation [26]. For the P type silicon wafer, the largest piezoresistive coefficient is emerged along the crystal direction [ $\left[\begin{array}{lll}1 & 1 & 0\end{array}\right]$. Therefore, in the design, all

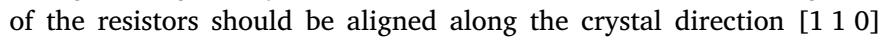
[27]. In this case, the relationship between resistance variation and stress can be expressed by Eq. (5) [28]:

$$
\begin{aligned}
\frac{\Delta R}{R} & =\frac{1}{2}\left(\pi_{11}+\pi_{12}+\pi_{44}\right) \sigma_{l}+\frac{1}{2}\left(\pi_{11}+\pi_{12}-\pi_{44}\right) \sigma_{t} \\
& =\frac{1}{2}\left(\pi_{11}+\pi_{12}\right)\left(\sigma_{l}+\sigma_{t}\right)+\frac{1}{2} \pi_{44}\left(\sigma_{l}-\sigma_{t}\right)
\end{aligned}
$$

where $\sigma_{l}$ and $\sigma_{t}$ are the longitudinal stress and the transverse stress respectively. $\pi_{11}, \pi_{12}$, and $\pi_{44}$ are the piezoresistive coefficients whose values under low doping concentration at room temperature are listed in Table 1. For P type silicon, $\pi_{44}$ is far larger than $\pi_{11}$ and $\pi_{12}$, so the effect of $\pi_{11}$ and $\pi_{12}$ can be neglected in the calculation. To achieve a high sensitivity, piezoresistors should be located at the place where the stress difference between $\sigma_{l}$ and $\sigma_{t}$ reaches the maximum.

The mechanical stress calculated by simulation should be converted into voltage in such a way that the stress value can be used to predict the equivalent output electrical signal [29]. In this case, the relation ship between output voltage and stress can be obtained by Eq. (6) [30]:

$\frac{U_{\text {out }}}{U_{\text {in }}}=\frac{\pi_{l}\left(\sum_{i=1}^{n} \sigma_{\text {li }} \nu_{i}\right)+\pi_{t}\left(\sum_{i=1}^{n} \sigma_{t i} \nu_{i}\right)}{\sum_{i=1}^{n} v_{i}}$

where $U_{\text {out }}$ is the output voltage, $U_{\text {in }}$ is the input voltage, $i$ is the

Table 1

Piezoresistive coefficients under low doping concentration at room temperature [24].

\begin{tabular}{lllll}
\hline Material & $\rho(\Omega \cdot \mathrm{cm})$ & $\begin{array}{l}\pi_{11} 10^{11} \\
\left(\mathrm{~Pa}^{1}\right)\end{array}$ & $\begin{array}{l}\pi_{12} 10^{11} \\
\left(\mathrm{~Pa}^{1}\right)\end{array}$ & $\begin{array}{l}\pi_{44} 10^{11} \\
(\mathrm{~Pa}\end{array}$ \\
\hline $\begin{array}{llll}{ }^{1} \\
\text { P-type silicon }\end{array}$ & 7.8 & +6.6 & 1.1 & +138.1 \\
N-type silicon & 11.7 & 102.2 & +53.4 & 13.6 \\
\hline
\end{tabular}




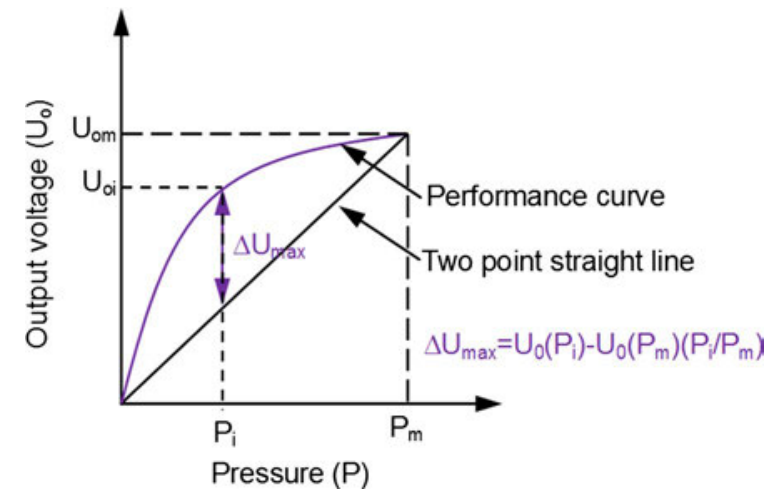

Fig. 1. Output voltage $V_{\mathrm{o}}$ versus pressure, $P$, and the corresponding two point straight line.

piezoresistive element number of the finite element model, $\sigma_{l i}$ and $\sigma_{t i}$ are the longitudinal and transverse stresses of the $i_{t h}$ piezoresistive element, respectively, and $\nu_{i}$ is the volume of the $i_{\text {th }}$ element on the piezoresistors. By this way, the simulation results can be applied to calculate the output of the sensor.

Silicon piezoresistive pressure sensors with thin membranes have to be sensitive, accurate, and stable during pressure measurement and maintain a linear relationship between the output voltage and pressure. Referring to the output voltage, $U_{\mathrm{o}}$, versus applied pressure, $P$, and the endpoint straight line shown in Fig.1, the nonlinearity $P N L_{i}$ of a pres sure sensor at a specific pressure $P_{\mathrm{i}}$ is defined as follows [31]:

$P N L_{i}=\frac{\Delta U_{\max }}{U_{o m}} \times 100 \%$

where $\Delta U_{\max }$ is the maximum output voltage difference between the real output and the idealized linear output (namely, the output along two point straight line), $U_{\text {om }}$ is the full scale output voltage at the maximum pressure input $P_{\mathrm{m}}$.

There are two reasons for non linearization of the pressure sensor, one is related to the excessive load on the membrane which directly destroys the linearity principle of the piezoresistive effect, and another is the oversized membrane deflection that changes linearity to non linearity in the relation between pressure and deformation. The case of excessive load on the membrane is not discussed in this work, after all, it belongs to an accident. Therefore, the case for oversized deflection will be the only one discussed here. When the deflection exceeds the definite value compared to the thickness of membrane, the large de flection theories work, and then, the linearity relationship between deflection and pressure will change to nonlinearity [32].

\subsection{Structural design}

A novel FBBM structure featuring a square bossed membrane with four short beams is designed to measure pressure less than $5 \mathrm{kPa}$. The sensor chip is fabricated with $\mathrm{N}$ type silicon wafer with the thickness of $300 \pm 5 \mu \mathrm{m}$. Four beams are located averagely on the bossed mem brane and they are perpendicular to the middle of each pedestal side. The FBBM structure is constructed in detail as shown in Fig. 2a, where $L$ is the effective side length of the membrane, $a, b$ and $h$ are the length, width and thickness of the beam, respectively. The rearview of the proposed structure is presented in Fig. 2b, where $H$ represents the thickness of membrane, $w$ and $t$ are the side length and thickness of the central mass. The dorsal cavity fabricated by wet etching provides space for deformation of the membrane, and a $54.7^{\circ}$ inclined wall is appeared due to anisotropy of single crystal silicon [24]. In this model, SCRs are expected to be located at the joint between each beam and membrane pedestal side. Moreover, the central mass is expected to play an im portant role in local stiffening, it is also beneficial to limit a large de flection effect and thereby reduces the mechanical nonlinearity error.

\subsection{Geometry analysis}

The performances of the proposed sensor chip are calculated by non linear static analysis and modal analysis by the commercially available finite element analysis (FEA) software COMSOL Multiphysics. Only a quarter of the finite element model is established for the sensor chip due to the symmetry as shown in Fig. 3a. In accordance with the previous discussion, the strain energy is mainly concentrated at the edge of the SCRs as indicated by the red area. This kind of SCRs con dition benefits from the novel stress concentration enhanced structure. Fig. $3 \mathrm{~b}$ reflects the longitudinal direction stress distribution along $\mathrm{x}$ path from one edge to another under $5 \mathrm{kPa}$ uniform pressure and il lustrates the relationship between $\mathrm{x}$ path and stress. It can be observed that the maximum stress along $x$ path appears at SCRs, and the stress in the region of the central mass is almost equal to zero. Owing to the stiffness change created by the novel beams structure combined with a central mass, the strain energy is strictly limited in a narrow area on the surface of the beams and the energy is not dispersed easily outside the SCRs. So the strain energy utilized by four piezoresistors is more than flat membrane, which makes it possible to obtain even higher sensi tivity. Based on the small deformation theory introduced above, the central mass pattern can make a contribution to the stiffness change, which is possible to decrease the pressure nonlinearity to smaller than $1.0 \%$ FSS. By this means, the situation of simultaneously improving sensitivity and linearity will be happened for the proposed structure.

\subsection{Geometry optimization}

In some degree the sensitivity of the pressure sensors rely on the membrane structure, which in turn is dependent on the geometry di mensions of the membrane. To optimize the geometry structure and determine the structural dimensions, it is necessary to deduce the re lationship between the structure and stress for FBBM sensor design. The simulation results of the longitudinal stress and deflection with dif ferent structural dimension variables are presented below. On the basis of the formulas of traditional $C$ type membrane, the maximum stress and deflection of the membrane are the power functions of each single structural dimension variable [33]. Theoretically, the function re lationship between FBBM structure and $C$ type should be similar. Based on Yu's study [12], Young's elastic modulus $E$ had almost no influence on the stress $\sigma$, but revealed the inversely proportional relationship to the deflection $\omega$. As a result, the formulas of FBBM are expressed by Eqs. (8) and (9):

$\sigma=Q_{1} \cdot P \cdot L^{i 1} \cdot H^{k 1} \cdot a^{m 1} \cdot b^{n 1} \cdot h^{r 1} \cdot w^{s 1} \cdot t^{z 1}$

$\omega=Q_{2} \cdot P \cdot E^{-1} \cdot L^{i 2} \cdot H^{k 2} \cdot a^{m 2} \cdot b^{n 2} \cdot h^{r 2} \cdot w^{s 2} \cdot t^{z 2}$

where $L, H, a, b, h, w, t$ are the structural dimensions as seen in Fig.1; $\sigma$ and $\omega$ are the maximum longitudinal stress and maximum deflection of the membrane under pressure; and $Q_{1}, Q_{2}, i_{1}, i_{2}, k_{1}, k_{2}, m_{1}, m_{2}, n_{1}, n_{2}, r_{1}$, $r_{2}, s_{1}, s_{2}, z_{1}, z_{2}$ are the coefficients. To calculate the coefficients in Eqs. (8) and (9), each single dimension variable should be discussed sepa rately. It means that other variables should be seen as constants when one variable is studied.

As shown in Fig.4, the stress and deflection are increased when the membrane length is added. The relationships between the FBBM per formance and membrane length should satisfy Eqs. (10) and (11):

$\sigma=Q_{1 l} \cdot L^{i 1}$

$\omega=Q_{2 l} \cdot L^{i 2}$

where $Q_{1 l}, Q_{2 l}$ are the sub coefficients of the variable $L$, and the other parameters have been previously mentioned. A series of $\sigma$ and $\omega$ are calculated by COMSOL numerical simulation when the membrane length is varied. Then, the Eqs. (10) and (11) are derived as follows by curve fitting method. 

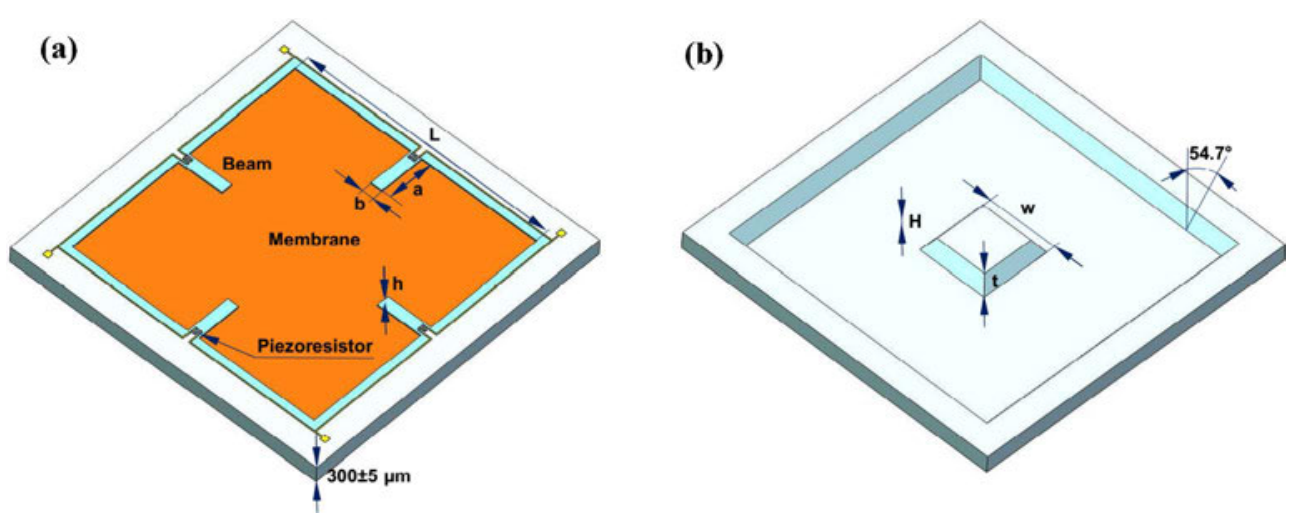

Fig. 2. (a) Sketch of the FBBM structure in the front view; (b) Rearview sketch of the FBBM structure.

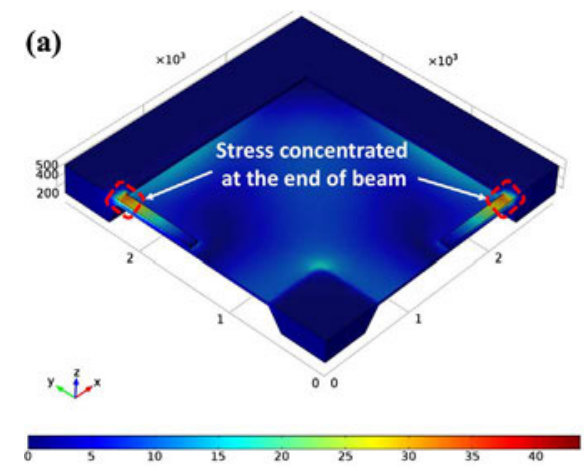

$\sigma=8.16818 \times 10^{-10} L^{2.93978}$

$\omega=8.93579 \times 10^{-19} L^{5.07637}$

In order to verify the goodness of fit between these equations and the simulation results, the residual curves of stress and deflection are also illustrated in Fig.4. The residual curve is drawn by residual error points that reflects the difference between the actual value of each point on the scatterplot and the predicted value of the regression equation. To achieve the best goodness of fit, the coefficient of determination $\left(R^{2}\right)$ and residual sum of squares (RSS) are introduced.

In statistics, the coefficient of determination, denoted $R^{2}$, is a number that indicates the proportion of the variance in the dependent variable that is predictable from the independent variable. $R^{2}$ is a sta tistic that will give the information about the goodness of fit of a model. In regression, the coefficient of determination $R^{2}$ is a statistical measure of how well the regression line approximates the real data points. An $R^{2}$ closer to 1 indicates that the regression line perfectly fits the data. RSS means the deviations predicted from actual empirical values of data. It

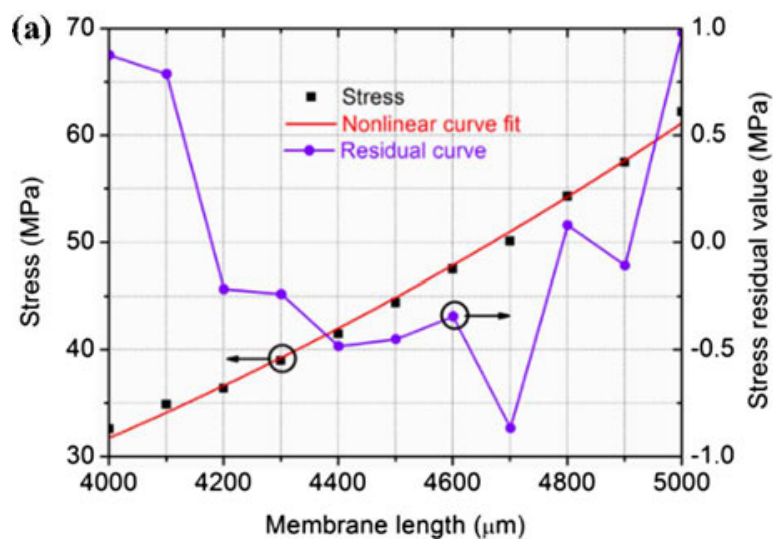

is a measure of the discrepancy between the data and an estimation model. A small RSS indicates a tight fit of the model to the data.

Based on the definition of $R^{2}$ and RSS, the stress $R 2 \sigma$ of Eq. (12) and deflection $R 2 \omega$ of Eq. (13) are equal to 0.99536 and 0.99996 , the $R S S_{\sigma}$ and $R S S_{\omega}$ are equal to 3.9514 and 0.0005 , respectively. The results indicate that the fitting equations and curves match well with the si mulation results.

Following the same analysis method, the relevant equations and curves to other several variables will be established easily. Adopt the method of combining all the equations related to each single variable, the equations of Eqs. (8) and (9) can be determined. The relative main equations are shown as follows:

$$
\begin{aligned}
& \sigma=Q_{1} \frac{P L^{2.93978} a^{0.31799}}{H^{1.90014} b^{0.3945} h^{0.40149} w^{0.21938} t^{0.02068}} \\
& w=Q_{2} \frac{P L^{5.07637}}{E H^{2.35753} a^{0.16714} b^{0.2673} h^{0.5443} w^{0.73648} t^{0.09719}}
\end{aligned}
$$

Fig. 4. (a) Relationship between stress and membrane length; (b) Relationship between deflection and membrane length. 
Table 2

Curve fitting values of the FBBM variables.

\begin{tabular}{|c|c|c|c|c|c|}
\hline Eq. number & $R^{2}$ & RSS & Eq. number & $R^{2}$ of rRR determination $\mathrm{R}^{2}$ & RSS \\
\hline Stress related to $L$ & 0.99536 & 3.9514 & Deflection related to $b$ & 0.97546 & 0.05362 \\
\hline Deflection related to $L$ & 0.99996 & 0.0005 & Stress related to $h$ & 0.92392 & 21.26792 \\
\hline Stress related to $H$ & 0.97737 & 3.49524 & Deflection related to $h$ & 0.96750 & 0.07817 \\
\hline Deflection related to $H$ & 0.99892 & 0.04582 & Stress related to $w$ & 0.97745 & 0.86236 \\
\hline Stress related to $a$ & 0.99209 & 4.04136 & Deflection related to $w$ & 0.98947 & 0.02305 \\
\hline Deflection related to $a$ & 0.98820 & 0.01395 & Stress related to $t$ & 0.96888 & 0.405 \\
\hline Stress related to $b$ & 0.97160 & 25.92862 & Deflection related to $t$ & 0.93648 & 0.10868 \\
\hline
\end{tabular}

From Eqs. (14) and (15), with the increase of the membrane length $L$, the maximum stress and deflection, both, experience a rise, namely, improve sensitivity, but worsen linearity. Meanwhile, the impacts for the membrane thickness $H$, beam width $b$, beam thickness $h$, central mass width $w$ and central mass thickness $t$ on the mechanical perfor mance are consistent except the beam length $a$. Unlike the other vari ables, the beam length is the only variable which is in directly pro portional to the stress and inversely proportional to the deflection. It indicates that it is possible to synchronously improve the sensitivity and linearity when the beam length is chosen appropriately. Thus, it is hopeful to alleviate the contradiction between sensitivity and linearity when proper dimensions are determined. It is worth noting that the indexes of the $L$ and $H$ are larger than other variables, which means $L$ and $H$ are more sensitive in influencing the performance of the sensor, so the accuracy for fabricating these two variables should be higher.

In order to prove the rationality and accuracy of the hypothesis about functional forms, the values of the coefficient of determination $R^{2}$ and residual sum of squares RSS are listed in Table 2. The values of $R^{2}$ and RSS demonstrate that the good curves fitting have been achieved for the corresponding equations except Eq. "Stress related to $b$ " and Eq. "Stress related to $h$ ", whose residual sum of squares RSS for beam width and beam thickness are a little big. This is because the correlations between these two dimension variables and stress are not high, which leads to a strong undulation for the stress fitting curve. Overall, how ever, the results certify the goodness of fit.

Fig.5 shows the impacts of structural dimension variables on the stress. It can be regarded as an estimation for the performance of the sensor, as each dimension variable has different effect on the stress. An intersection line is also introduced in this figure. For clear observation of the relationships between different variables, the partially enlarged figures of each structural dimension variable except membrane length are drawn in Fig.6. By a comprehensive consideration, a series of structural dimension variables are determined based on crossover

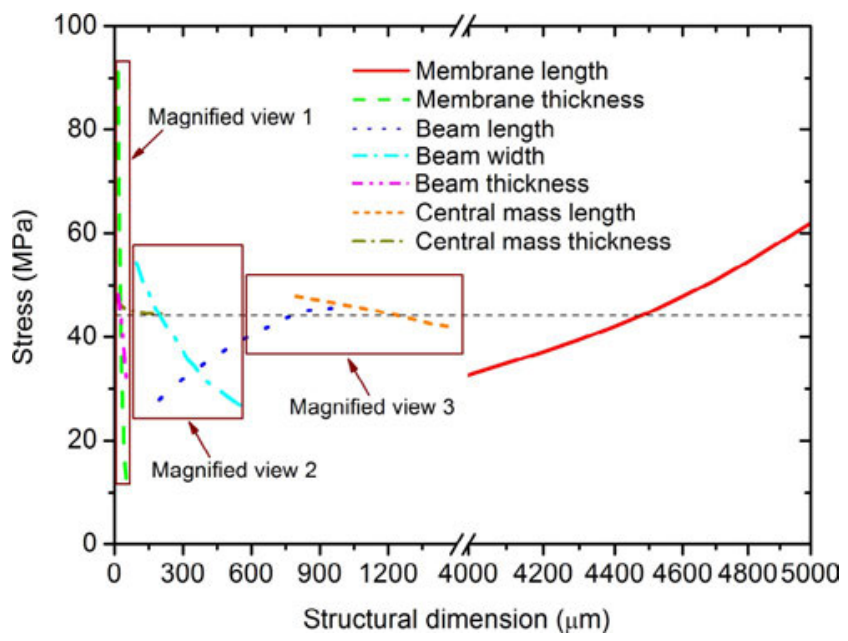

Fig. 5. Stress versus structural dimension variables. points between variable curves and intersection line as listed in Table 3.

On the account of the parameters for the FBBM structure presented in Table 3, the simulation results related to the stress and deflection are obtained as follows: $\sigma$ is equal to $43.6 \mathrm{MPa}$, and $\omega$ is equal to $3.14 \mu \mathrm{m}$. Put the above determined dada, Young's elastic modulus $\left(1.9 \times 10^{11} \mathrm{~N} / \mathrm{m}^{2}\right)$ and pressure (5 kPa) into Eqs. (14) and (15), $Q_{1}$ and $Q_{2}$ are equal to $1.817 \times 10^{-3}$ and $4.681 \times 10^{-4}$, respectively. Finally, the main stress and deflection equations specific to FBBM are expressed as:

$$
\begin{aligned}
& \sigma=1.817 \times 10^{-3} \frac{P L^{2.93978} a^{0.31799}}{H^{1.90014} b^{0.3945} h^{0.40149} w^{0.21938} t^{0.02068}} \\
& w=4.681 \times 10^{-4} \frac{P L^{5.07637}}{E H^{2.35753} a^{0.16714} b^{0.2673} h^{0.5443} w^{0.73648} t^{0.09719}}
\end{aligned}
$$

From Eqs. (16) to (17), the stress and deflection of the FBBM re garding the structural dimension variables are clearly presented. The design and fabrication processes can be executed more efficiently as long as the dimension variables are determined in advance. Yet the ranges of all the variables discussed are constrained by actual demands and processing level, and it means the equations are effective in certain scopes.

To theoretically compute the sensitivity of the proposed sensor, the longitudinal stress $\sigma_{x}$ and transversal stress $\sigma_{y}$ along the path are presented in Fig.7. By comparing the results of the two sets of data, the stress $\sigma_{x}$ is larger than $\sigma_{y}$. It is indicated that the piezoresistors implanted on the silicon substrate should be parallel to the $\mathrm{x}$ axis of the membrane. This can maximize the utilization of the transverse effect and decrease the longitudinal effect [27]. Then, a high output voltage can be achieved. Besides, it is found that the SCRs are located at the edge between each beam and membrane pedestal side as we expected. Piezoresistors should be placed on the SCRs because more strain energy can be utilized, and therefore the piezoresistive sensi tivity can be enhanced definitely. Through the comprehensive ana lysis from the Eq. (6), the output voltage of the sensor for a certain mechanical pressure $P(5 \mathrm{kPa})$ and input voltage $U_{\text {in }}(5 \mathrm{~V})$ is then calculated by Eq. (18):

$S=\frac{\Delta U}{U_{\text {in }}} \frac{1}{P}=\frac{0.345 \times 10^{-5}(\sigma x-\sigma y)_{5 k P a}}{U_{\text {in }}} \frac{1}{P}$

Eventually, the sensitivity of $4.83 \mathrm{mV} / \mathrm{V} / \mathrm{kPa}$ can be deduced.

\section{Fabrication and measurement}

Four inch $(100 \pm 0.5 \mathrm{~mm}) \mathrm{N}$ type silicon wafer oriented in the ( $\left.\begin{array}{lll}1 & 0 & 0\end{array}\right)$ direction with a resistivity of $10 \Omega \mathrm{cm}$ are used as substrate of the FBBM chip. They are $300 \pm 5 \mu \mathrm{m}$ thickness and double sides po lished with a total thickness variation of $<5 \mu \mathrm{m}$. The specific fabrica tion processes are illustrated in Fig. 8. (a) The first step in fabrication is to grow a $500 \pm 20 \mathrm{~nm}$ silicon oxide on both sides of the substrate by thermal oxidation. This oxide layer is used to isolate the metallization from the silicon substrate; (b) then, lithography is performed on the front side of silicon oxide to pattern piezoresistors. $\mathrm{P}$ type resistors and heavy doping of contact regions are formed by ion implantation of 

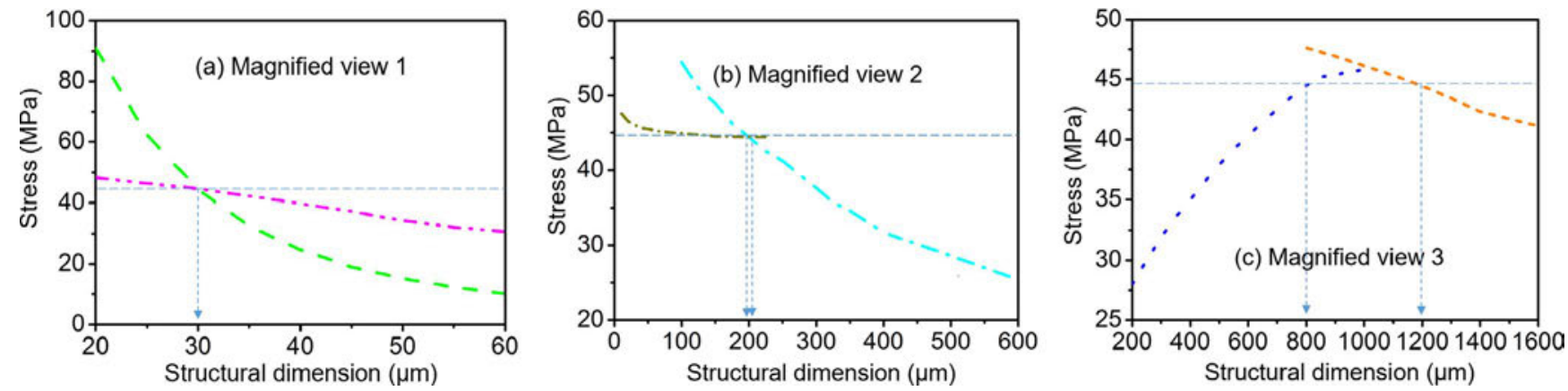

Fig. 6. Partially enlarged diagrams of structural dimension curves.

Table 3

Parameters of the FBBM membrane.

\begin{tabular}{llllllll}
\hline Parameter & $L$ & $H$ & $a$ & $b$ & $h$ & $w$ & $t$ \\
\hline Dimension $(\mu \mathrm{m})$ & 4500 & 30 & 800 & 200 & 30 & 1200 & 220 \\
\hline
\end{tabular}

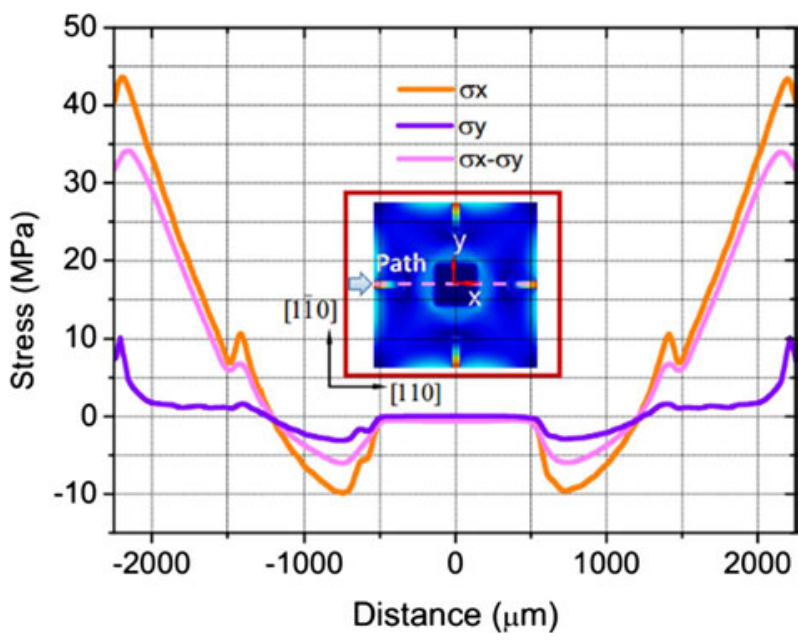

Fig. 7. Stress distribution curve of the path.

boron; (c) subsequently, the low pressure chemical vapor deposition (LPCVD) is adopted to grow the passivation layers of $\mathrm{Si}_{3} \mathrm{~N}_{4}$ to protect the piezoresistors; (d) in the following, $\mathrm{SiO}_{2}$ layers are grown again by

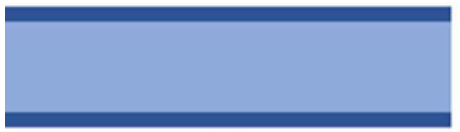

(a)

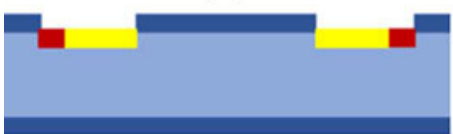

(b)

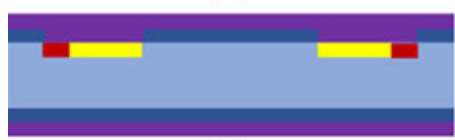

(c)

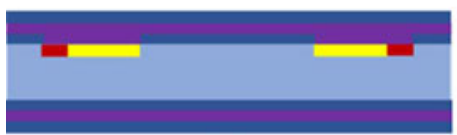

(d)

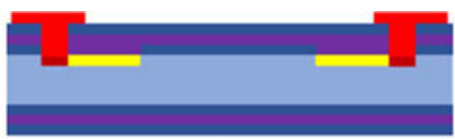

(e)

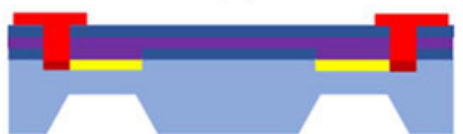

(f)

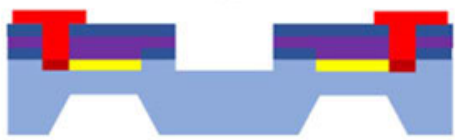

(g)

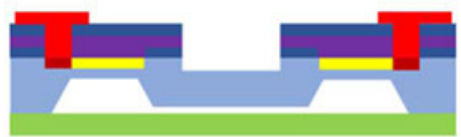

(h) the plasma enhanced chemical vapor deposition (PECVD) to further improve insulation; (e) after that, the reactive ion etching (RIE) process is used to etch the ohm contact regions between the piezoresistors and the metal lead. The e beam evaporation deposition is performed to sputter $\mathrm{Al}$ for the purpose of connecting of resistors and forming of bonding pads; (f) with the objective of creating a cavity and a central mass for producing the pressure sensitive membrane, $\mathrm{KOH}$ wet etching process is used to deeply etch the bottom of the silicon wafer. Due to anisotropy of single crystal silicon, a $54.7^{\circ}$ inclined wall is left after deep etching; ( $\mathrm{g}$ ) afterwards, the RIE is applied again to produce beams of the FBBM structure on the front side of the silicon wafer; (h) in order to obtain an absolute pressure reference chamber, the bottom side of the wafer is attached to the Pyrex 7740 glass by the anodic bonding process under vacuum condition. A microphotograph of the finished FBBM sensor chip is presented in Fig. 9.

The packaging and interconnecting structure of the FBBM sensor are shown in Fig. 10(a). The metal material of the external structure is 304 stainless steel. In order to improve the sealing and insulation of the sensor, the epoxy adhesive (60\% ethoxyline resin, $30 \%$ quartz powder, $10 \%$ dioctyl phthalate) is filled in the shell and solidified for $24 \mathrm{~h}$ at room temperature. The wire connecting the sensor and aviation plug is polytetrafluoroethylene aviation cable, which can resist high tempera ture more than $200^{\circ} \mathrm{C}$. The process of the packaged sensor is presented in Fig. 10(b). To protect the welding spot, the fluororubber heat shrink tube is covered at the connection between every two wires. Between the stainless steel shell and fixing ring, laser welding is chosen for con nection, which can improve the long term stability and application of the sensor.

The schematic diagram for testing is shown in Fig.11. Before the measurement, connect the equipment and turn the ball valve

Fig. 8. Main fabrication processes used to manufacture the FBBM sensor chip. 


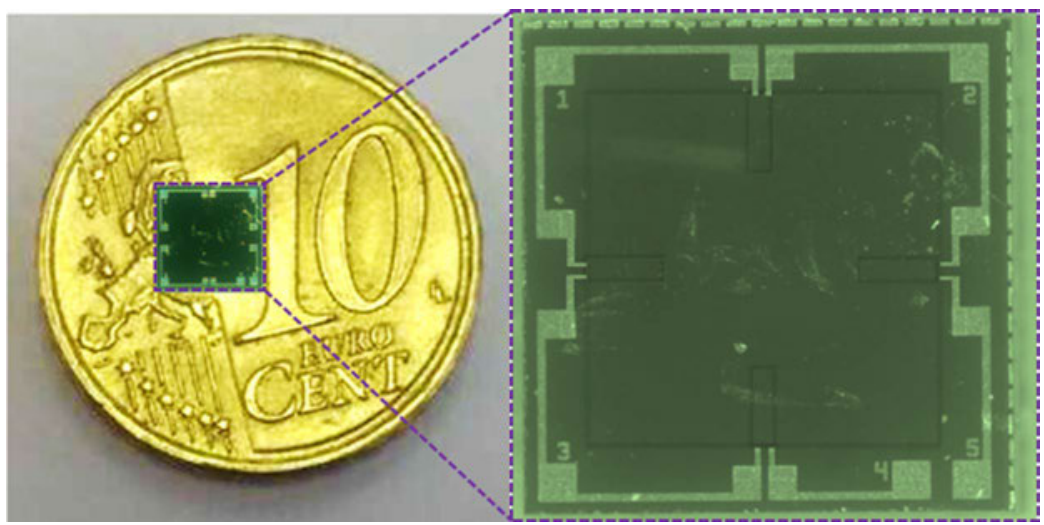

Fig. 9. The microphotograph of the FBBM sensor chip.

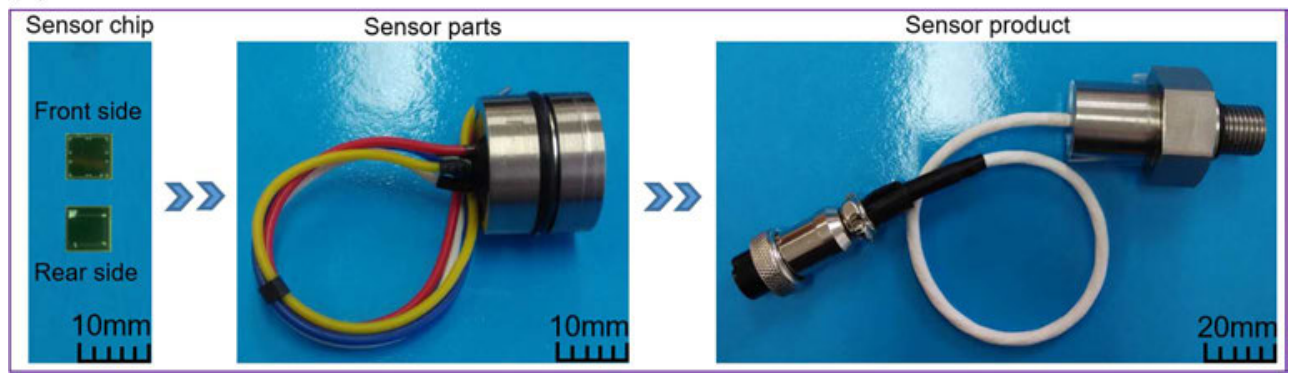

Fig. 10. Assembly diagram of the sensor, (a) the

(a)

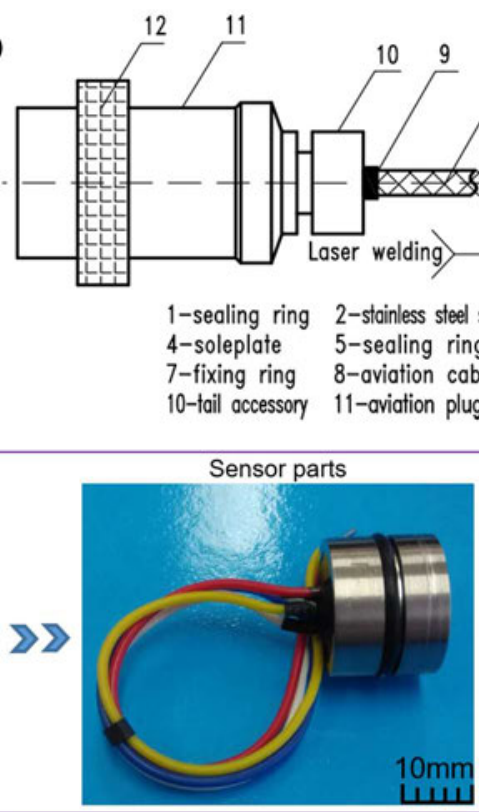

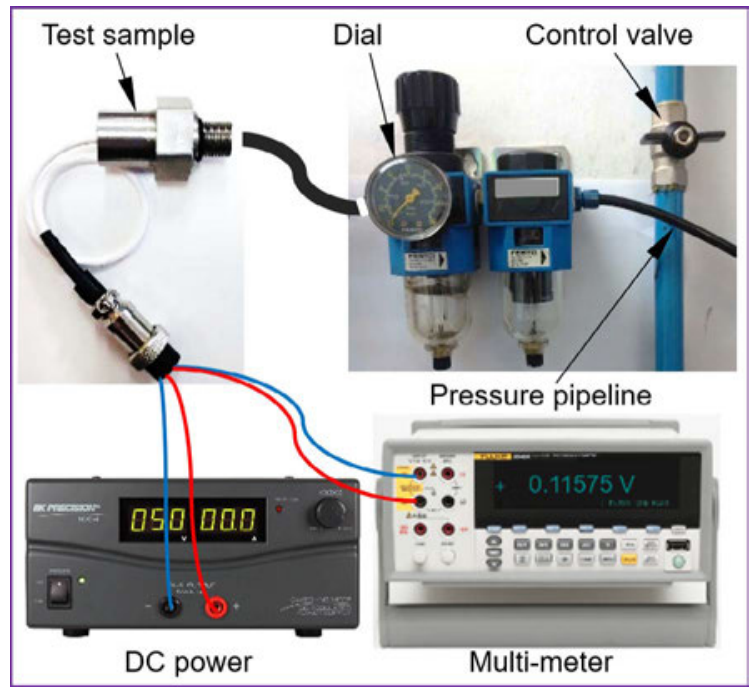

Fig. 11. Experimental schematic diagram for testing.

anticlockwise. By adjusting the control valve, the target pressure value for test can be obtained. In order to ensure the airtightness of the measurement, the joint between the sensor port and connecting tube should be twined several laps using the rubber belt. A constant voltage

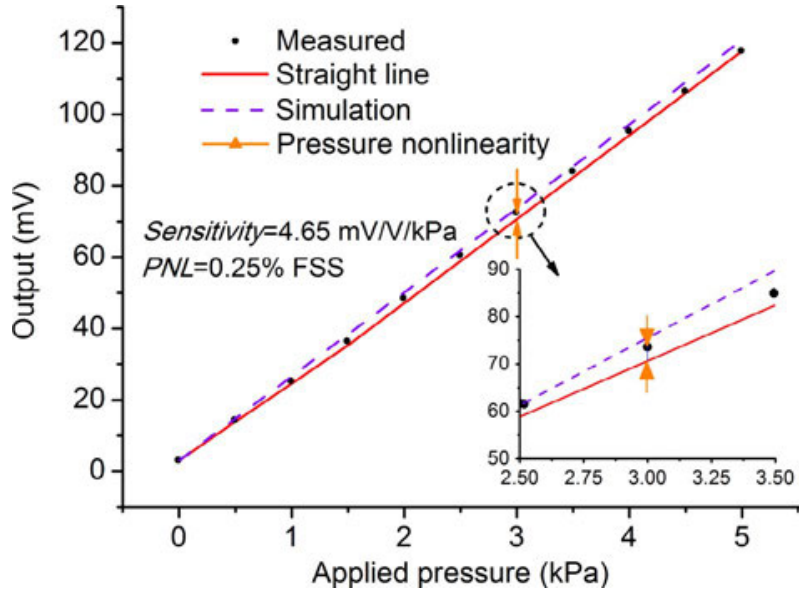

Fig. 12. Graph of the output voltage versus applied pressure.

of $5 \mathrm{~V}$ is provided to the Wheatstone bridge of the sensor using a High Current Switching DC Power Supplies (Model BK1694). The output of the sensor at different pressure loads is read by a digital multimeter (FLUKE 8845A). 
Table 4

Static performance of the sensor at room temperature.

\begin{tabular}{ll}
\hline Parameter & Value \\
\hline Full range pressure (kPa) & 5 \\
Supply voltage (V) & 5 \\
Full range output (mV) & 119.2 \\
Sensitivity (mV/V/kPa) & 4.65 \\
PNL (\% FSS) & 0.25 \\
Repeatability (\%FSS) & 0.19 \\
Pressure Hysteresis (\%FSS) & 0.14 \\
Accuracy (\%FSS) & 0.34 \\
\hline
\end{tabular}

Table 5

Comparison with other pressure sensors.

\begin{tabular}{llll}
\hline Sensor & $\begin{array}{l}\text { Sensitivity } \\
(\mathrm{mV} / \mathrm{V} / \mathrm{kPa})\end{array}$ & $\begin{array}{l}\text { Pressure nonlinearity } \\
(\% \mathrm{FSS})\end{array}$ & $\begin{array}{l}\text { Accuracy } \\
\text { (\% FSS) }\end{array}$ \\
\hline FBBM sensor & 4.65 & 0.25 & 0.34 \\
Sensor in [13] & 1.47 & 0.19 & 0.24 \\
Sensor in [17] & 1.75 & 0.05 & 0.68 \\
Sensor in [33] & 3.68 & 0.36 & - \\
\hline
\end{tabular}

\section{Results and discussion}

\subsection{Experimental results}

The measured results are drawn in Fig. 12. The graph depicts the output voltage of the sensor as a function of the pressure varies from 0 to $5 \mathrm{kPa}$ at room temperature $\left(25^{\circ} \mathrm{C}\right)$. Meanwhile, the straight line is depicted for calculating pressure nonlinearity. In this figure, the sen sitivity and calculated pressure nonlinearity are $4.65 \mathrm{mV} / \mathrm{V} / \mathrm{kPa}$ and $0.25 \%$ FSS, which indicates a good agreement between the actual measurement and simulation results. Besides, the detailed technical data of the sensor is listed in Table 4.

\subsection{Discussion}

The FBBM sensor demonstrates that it does has the capacity of al leviating the contradiction between sensitivity and linearity to realize the micro measurement with high accuracy as shown in Table 5. Compared with the other three reported sensors, the sensitivity of the FBBM sensor is the largest, which is more than two times bigger than that reported in [13]. At the same time, the pressure nonlinearity for the FBBM is intermediate among the four types of sensors, unlike [17] where a small pressure nonlinearity is obtained but its sensitivity is too low. Thus, it can be concluded that the FBBM sensor achieves a high sensitivity and a low pressure nonlinearity when compared with the reported sensors.

According to the design results and analyses, the novel structural pressure sensors are fabricated and assembled, and the overall perfor mances are also verified. It is confirmed from the experimental results that the actual sensitivity is similar to the estimated sensitivity, which proves the validity of the design process. However, there is still a de viation of 3.9\% between the simulated result and experimental result as shown in Fig. 11. The reasons mainly come from the following three aspects. One results from the poor consistency of the four piezoresistors in the Wheatstone bridge due to the inconsistent dopant concentration of the resistors involved in the process of ion implantation. Besides, the residual stresses on $\mathrm{Si}_{3} \mathrm{~N}_{4}$ and $\mathrm{SiO}_{2}$ passivation layers may also con tribute to the relatively poor accuracy [34]. Finally, the errors in the bulk and surface microfabrication can't be avoided, so it is not possible to obtain a theoretical performance after many manufacture proce dures.

\section{Conclusions}

In this study, a novel structural FBBM enhancing the sensitivity and linearity of a silicon piezoresistive pressure sensor was proposed. A series of models for deducing the relative equations for these structures were established based on the simulation results. According to the curve fitting, the final relationship between the mechanical perfor mance and dimension variables was determined, which could provide a guideline for designing the sensor with the FBBM structure. By altering the values in the equations, the optimized dimensions for the mem brane could be found. This method was useful to accurately design and fabricate the FBBM pressure sensor.

The performance of the FBBM was carried out by practical experi ments. The results showed that, when the applied pressure was $5 \mathrm{kPa}$, the sensor achieved the sensitivity of $4.65 \mathrm{mV} / \mathrm{V} / \mathrm{kPa}$ and pressure nonlinearity of $0.25 \%$ FSS, and thereby the contradiction between sensitivity and linearity was alleviated. In terms of micro size, accuracy and reliability, the FBBM sensor obtained a good performance, so it was a proper choice for measuring micro pressure less than $5 \mathrm{kPa}$.

\section{Acknowledgments}

The work was supported by the China Scholarship Council for studying abroad (201508320275) and Erasmus + project (2015 1 ES01 KA107 015460). We also would like to thanks for the help in microfabrication from Suzhou Institute of Nano Tech and Nano Bionics (SINANO), Chinese Academy of Sciences.

\section{Conflict of interest}

The authors declare no conflict of interest.

\section{References}

[1] Kumar SS, Pant BD. Design principles and considerations for the "ideal" silicon piezoresistive pressure sensor: a focused review. Microsyst Technol 2014;20:1213-47. http://dx.doi.org/10.1007/s00542-014-2215-7.

[2] Gkotsis P, Lara-Castro M, López-Huerta F, Herrera-May AL, Raskin J-P. Mechanical characterization and modelling of Lorentz force based MEMS magnetic field sensors. Solid State Electron 2015;112:68-77. http://dx.doi.org/10.1016/j.sse.2015.02.004.

[3] Shaby SM, Premi MSG, Martin B. Enhancing the performance of MEMS piezoresistive pressure sensor using germanium nanowire. Procedia Mater Sci 2015;10:254-62. http://dx.doi.org/10.1016/j.mspro.2015.06.048.

[4] Kumar SS, Pant BD. Effect of piezoresistor configuration on output characteristics of piezoresistive pressure sensor: an experimental study. Microsyst Technol 2016;22:709-19. http://dx.doi.org/10.1007/s00542-015-2451-5.

[5] Lin C-Y, Chiou J-C. Design and fabrication of MEMS-based thermally-actuated image stabilizer for cell phone camera. Solid State Electron 2012;77:64-71. http:// dx.doi.org/10.1016/j.sse.2012.05.013.

[6] Ho J-J. The design and fabrication of a micro-thermal/pressure-sensor for medical electro-skin application. Solid State Electron 2002;46:1205-9. http://dx.doi.org/ 10.1016/S0038-1101(02)00012-6.

[7] Samaun, Wise KD, Angell JB. An IC piezoresistive pressure sensor for biomedical instrumentation. IEEE Trans Biomed Eng 1973;BME-20:101-9. http://dx.doi.org/ 10.1109/TBME.1973.32417.

[8] Rajavelu M, Sivakumar D, Joseph Daniel R, Sumangala K. Perforated diaphragms employed piezoresistive MEMS pressure sensor for sensitivity enhancement in gas flow measurement. Flow Meas Instrum 2014;35:63-75. http://dx.doi.org/10.1016/ j.flowmeasinst.2013.12.004.

[9] Aravamudhan S, Bhansali S. Reinforced piezoresistive pressure sensor for ocean depth measurements. Sens Actuators A Phys 2008;142:111-7. http://dx.doi.org/10. 1016/j.sna.2007.04.036.

[10] Shimazoe M, Matsuoka Y, Yasukawa A, Tanabe M. A special silicon diaphragm pressure sensor with high output and high accuracy. Sens Actuators 1982;2:275-82. http://dx.doi.org/10.1016/0250-6874(81)80047-9.

[11] Marco S, Samitier J, Ruiz O, Morante JR, Esteve J. High-performance piezoresistive pressure sensors for biomedical applications using very thin structured membranes. Meas Sci Technol 1996;7:1195-203. http://dx.doi.org/10.1088/0957-0233/7/9/ 002.

[12] Yu Z, Zhao Y, Sun L, Tian B, Jiang Z. Incorporation of beams into bossed diaphragm for a high sensitivity and overload micro pressure sensor. Rev Sci Instrum 2013;84:015004. http://dx.doi.org/10.1063/1.4775603.

[13] Tian B, Zhao Y, Jiang Z. The novel structural design for pressure sensors. Sens Rev 2010;30:305-13. http://dx.doi.org/10.1108/02602281011072189.

[14] Albert Chiou J, Chen S. Pressure nonlinearity of micromachined piezoresistive 
pressure sensors with thin diaphragms under high residual stresses. Sens Actuators A Phys 2008;147:332-9. http://dx.doi.org/10.1016/j.sna. 2008.03.012.

[15] Kinnell PK, King J, Lester M, Craddock R. A hollow stiffening structure for lowpressure sensors. Sens Actuators A Phys 2010;160:35-41. http://dx.doi.org/10. 1016/j.sna.2010.03.024.

[16] Matsuoka Y, Yamamoto Y, Tanabe M, Sshimada S, Yamada K, Yasukawa A, et al. Low-pressure measurement limits for silicon piezoresistive circular diaphragm sensors. J Micromech Microeng 1995;5:32-5. http://dx.doi.org/10.1088/0960$1317 / 5 / 1 / 006$.

[17] Sandmaier H, Kuhl K. A square-diaphragm piezoresistive pressure sensor with a rectangular central boss for low-pressure ranges. IEEE Trans Electron Devices 1993;40:1754-9. http://dx.doi.org/10.1109/16.277331.

[18] Pramanik C, Saha H. Piezoresistive pressure sensing by porous silicon membrane. IEEE Sens J 2006;6:301-9. http://dx.doi.org/10.1109/JSEN.2006.870171.

[19] English JM, Allen MG. Wireless micromachined ceramic pressure sensors. In: Tech Dig IEEE Int MEMS 99 Conf Twelfth IEEE Int Conf Micro Electro Mech Syst (Cat No99CH36291); 1999. http://dx.doi.org/10.1109/MEMSYS.\%201999.746881.

[20] Wang L, Li J. A piezoresistive flounder element based on conductive polymer composite. Sens Actuators A Phys 2014;216:214-22. http://dx.doi.org/10.1016/j. sna.2014.05.010.

[21] Fraga MA, Furlan H, Massi M, Oliveira IC, Koberstein LL. Fabrication and characterization of a $\mathrm{SiC} / \mathrm{SiO}_{2} / \mathrm{Si}$ piezoresistive pressure sensor. Procedia Eng 2010;5:609-12. http://dx.doi.org/10.1016/j.proeng.2010.09.183.

[22] Zhang X, Wang E, Yang X. Design and simulation of Nano-diamond film pressure sensor. Vacuum 2014;99:189-91. http://dx.doi.org/10.1016/j.vacuum.2013.05. 023.

[23] Zhang S, Wang T, Lou L, Tsang WM, Sawada R, Kwong DL, et al. Annularly grooved diaphragm pressure sensor with embedded silicon nanowires for low pressure application. J Microelectromech Syst 2014;23:1396-407. http://dx.doi.org/10.1109/ JMEMS.2014.2313635.

[24] Hsu TR. MEMS and microsystems: design, manufacture, and nanoscale engineering. Wiley; 2008.

[25] Timoshenko S, Woinowsky-Krieger S. Theory of plates and shells. Tokyo: McGrawHill Kogakusha; 1959.

[26] Rochette F, Cassé M, Mouis M, Haziot A, Pioger T, Ghibaudo G, et al. Piezoresistance effect of strained and unstrained fully-depleted silicon-on-insulator MOSFETs integrating a $\mathrm{HfO}_{2}$ /TiN gate stack. Solid State Electron 2009;53:392-6. http://dx.doi.org/10.1016/j.sse.2009.01.017.

[27] Niu Z, Zhao Y, Tian B. Design optimization of high pressure and high temperature piezoresistive pressure sensor for high sensitivity. Rev Sci Instrum 2014;85:015001. http://dx.doi.org/10.1063/1.4856455.

[28] Song JW, Lee J-S, An J-E, Park CG. Design of a MEMS piezoresistive differential pressure sensor with small thermal hysteresis for air data modules. Rev Sci Instrum 2015;86:065003. http://dx.doi.org/10.1063/1.4921862.

[29] Martin B, Juliet V, Sankaranarayanan PE, Gopal A, Rajkumar I. Wireless implementation of mems accelerometer to detect red palm weevil on palms. In: 2013 Int Conf Adv Electron Syst; 2013. p. 248-52. http://dx.doi.org/10.1109/ICAES. 2013.6659402.

[30] Chen L-T, Chang J-S, Hsu C-Y, Cheng W-H. Fabrication and performance of MEMSbased pressure sensor packages using patterned ultra-thick photoresists. Sensors (Basel) 2009;9:6200-18. http://dx.doi.org/10.3390/s 90806200.

[31] Young WC, Budynas RG. Roark's formulas for stress and strain. New York: McGrawHill; 1986.

[32] Li C, Cordovilla F, Jagdheesh R, Ocaña JL. Design and optimization of a novel structural MEMS piezoresistive pressure sensor. Microsyst Technol 2016:1-11. http://dx.doi.org/10.1007/s00542-016-3187-6.

[33] Huang X, Zhang D. A high sensitivity and high linearity pressure sensor based on a peninsula-structured diaphragm for low-pressure ranges. Sens Actuators A Phys 2014;216:176-89. http://dx.doi.org/10.1016/j.sna. 2014.05.031.

[34] Meng X, Zhao Y. The design and optimization of a highly sensitive and overloadresistant piezoresistive pressure sensor. Sensors (Switzerland) 2016;16. http://dx. doi.org/10.3390/s1603034.

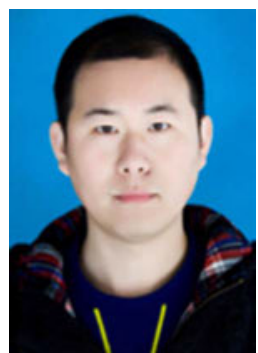

Chuang Li received the B.S. and M.S. degrees from School of Materials Science and Engineering, University of Jinan and School of Materials Science and Technology, Nanjing University of Aeronautics and Astronautics, China, in 2008 and 2011, respectively. He is currently working toward the Ph.D. degree in mechanical engineering in UPM Laser Centre, Polytechnical University of Madrid, Spain. His research interests include the design, fabrication, and characterization of MEMS sensors, especially the piezoresistive pressure sensor for low pressure measurement applications.

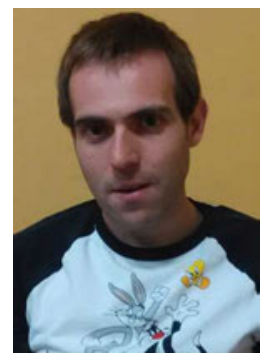

Francisco Cordovilla received B.S. in mechanical engineering and M.S. in laser technology both from the Polytechnical University of Madrid. He is currently developing his Ph.D degree about laser processing techniques in the UPM Laser Centre, Polytechnical University of Madrid, Spain, where he has published different works about problems concerning the physics of the industrial laser processes. His main research interest lies on simulation tools, specially based on the finite element method, to understand the physics associated to the laser processing of materials.

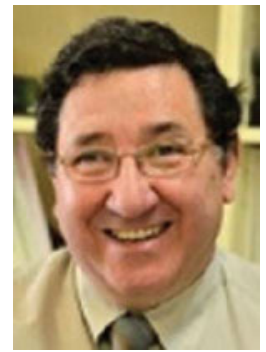

Jose L. Ocaña was awarded his MSc (1979) and a PhD (1982) in Industrial Engineering (Energy) at the Polytechnical University of Madrid (Spain). He is Chair Professor of Mechanical Engineering at the ETSII-UPM School of Engineering and Director of the UPM Laser Centre at this University. He is responsible for coordination of worldwide R \& D initiatives in the field of scientific and industrial applications of high-power lasers, especially in high-intensity laser matter interaction, laser welding, laser surface treatments, laser micromachining, and on-line monitoring and control of industrial laser applications. 